\section{Kidney \\ Blood Pressure \\ Research}

Kidney Blood Press Res 2013;37:116-123

DOI: 10.1159/000350065

Published onlıne: April 13, 2013

(c) 2013 S. Karger AG, Basel

www.karger.com/kbr

1423-0143/13/0373-0116\$38.00/0

This is an Open Access article licensed under the terms of the Creative Commons Attribution-

NonCommercial-NoDerivs 3.0 License (http://www.karger.com/OA-license), applicable to the

online version of the article only. Distribution for non-commercial purposes only.

\title{
Significance of Urine Diagnostic Tests After Renal Transplantation
}

\author{
Fabienne Jenni Sebastian Riethmüller ${ }^{*}$ Rudolf P. Wüthrich \\ Division of Nephrology, University Hospital, Zurich, Switzerland; " Both authors contributed equally to \\ this work
}

\section{Key Words}

Kidney transplantation $\cdot$ Urinalysis $\cdot$ Urine osmolality $\cdot$ Osmolaluria $•$ Urinary tract infection • Bacteriuria

\begin{abstract}
Background: Validity, reliability and clinical value of classical urinary parameters for transplant monitoring are controversial. Urinary parameters were analyzed regarding cost-effectiveness, frequency of urinary tract infection and prediction of renal graft function and rejection. Methods: Urinary parameters of the first two postoperative weeks of 120 renal transplant patients were retrospectively correlated with the postoperative course. Results: Creatinine levels were significantly different on each postoperative day between the groups with and without rejection. Osmolaluria, diuresis and serum creatinine are equivalent in predicting graft rejection. Osmolaluria is not suitable as a distinguishing criterion between graft rejection and other complications. Measurement of glucosuria has no diagnostic value. Proteinuria has no prognostic relevance regarding rejection, although proteinuria $>0.5 \mathrm{~g} / \mathrm{l}$ occurred more often in patients with rejection. Despite antibiotic prophylaxis with co-trimoxazole, 41 of 120 patients (34\%) suffered from urinary tract infection (UTI; mostly E. coli) within the first 14 days after transplantation. Conclusions: The measurement of some classical urinary parameters delivers no diagnostic gain. UTIs are frequent despite antibiotic prophylaxis, but the use of urine cultures makes sense only if a (cheaper) semiquantitative test is positive.
\end{abstract}

Copyright (C) 2013 S. Karger AG, Basel

\section{Introduction}

Specific patterns in urinalysis provide information about renal diseases. Urine strip test allows semiquantitative evaluation of proteins, glucose, leucocytes and haemo-/myoglobin. Bacteriological evaluation, quantitative measurement of electrolytes and metabolites are further possibilities for urine diagnostic. Most renal transplants are functioning well 


\section{Kidney \\ Blood Pressure Research}

immediately after transplantation. In some cases function is delayed or does not occur at all. During the early phase (first 14 days) possible causes include ischemic and reperfusion injury, rejection and adverse drug effects. To maintain transplant function in case of rejection, early diagnosis and immediate therapy are crucial [1]. Even when clinically suspected, diagnosis is confirmed only histologically. Urine analysis might be a non-invasive and cost-efficient method for monitoring renal transplants. Previous studies reported that morphological features of urinary sediment including increasing cellularity, especially lymphocytes and tubular cells, could anticipate renal rejection [2]. The aim of our study was to evaluate urinary findings in the early phase after renal transplantation and to correlate them with acute rejection.

\section{Methods}

\section{Patients}

Between January 2007 and June 2008, 120 consecutive renal transplant patients were included and grouped according to the occurrence of histologically confirmed rejection in the first 14 days after transplantation.

\section{Methods}

24-hour urine collection was obtained daily to quantify diuresis, osmolality and osmolaluria. Twice a week, glucose and protein excretion was determined in the 24-hour urine collection. From spontaneous urine, strip test (Combur-Test ${ }^{\circledR}$, Roche Diagnostics, Mannheim, Germany) and microscopic examination as well as determination of osmolality, sodium, potassium, creatinine and protein were made Mondays and Thursdays. Every Thursday a urine culture took place.

Osmolality was quantified using the Fiske ${ }^{\circledast}$ Modell 210 Mikro-Osmometer (Advanced ${ }^{\circledR}$ Instruments Inc., Norwood, MA). Osmolaluria was calculated from 24-hour diuresis and osmolality. For strip test interpretation, Miditron Junior II ${ }^{\circledR}$ (Roche Diagnostics, Mannheim, Germany) was used. Serum creatinine was measured kinetic by the Jaffé method.

If results were normal distributed (Kolmogorow-Smirnow-test), mean values ( \pm standard deviation) and $t$-test were used, otherwise we used median values (interquartile range (IQR)) and Mann-Withney-U test. Null hypothesis was rejected when $\mathrm{p}<0.05$ (two tailed). Receiver-operating characteristic (ROC) curves were calculated for osmolality and osmolaluria [3]. Statistic calculation was performed with SPSS 17.0. (SPSS Inc, Chicago, IL)

\section{Results}

Table 1 shows the characteristics of the transplant recipients. From 120 patients, 95 (79\%) did not show acute rejection (NRej) in the first two postoperative weeks, whilst 25 (21\%) showed histological evidence for acute rejection (Rej). Twice as many men as women were transplanted.

Two thirds of all transplants were from donors after brain death, one third from living donors.

There were 100 donors of whom 53 were women. Average age approximately corresponded to the recipients and did not differ between living and post-mortem donors (52 \pm 15 years). Cold ischemia time of the organ was just about 90 min for living donor transplants and $716 \pm 241$ minutes for post-mortem renal transplants. Standard immunosuppression included prednisone, cyclosporine and mycophenolate without induction therapy. Of 38 biopsies, 25 showed evidence of acute rejection (24 patients Banff IIA, 1 patient Banff IIB), 15 in the first week and 10 in the second week. The other biopsies showed tubular necrosis or tubulointerstitial infiltrates not qualifying for Banff IA. No graft recipient died within the first 14 postoperative days. 


\section{Kidney Blood Pressure Research}

Table 1. Characteristics of transplant recipients

\begin{tabular}{lccc}
\hline & Total & NRej & Rej \\
\hline Number of patients & 120 & 95 & 25 \\
Age (years) & $51 \pm 12$ & $51 \pm 12$ & $51 \pm 13$ \\
Sex (m:f) & $87: 33$ & $72: 23$ & $15: 10$ \\
Renal diagnosis & & & \\
Chronic glomerulonephritis & 23 & 14 & 9 \\
Chronic pyelonephritis & 3 & 3 & 0 \\
Nephrosclerosis & 2 & 2 & 0 \\
Polycystic kidney disease & 17 & 16 & 1 \\
Diabetes mellitus type 1 & 13 & 12 & 1 \\
Diabetes mellitus type 2 & 6 & 4 & 2 \\
Hypertensive nephropathy & 3 & 2 & 1 \\
IgA nephropathy & 12 & 8 & 4 \\
Glomerulosclerosis & 4 & 2 & 2 \\
Renal insufficiency of unknown Aetiology & 9 & 9 & 0 \\
Others* & 28 & 23 & 5 \\
Type of donation & & & \\
Donors after brain death & 80 & 58 & 22 \\
Living donors & 40 & 37 & 3 \\
\hline * “Others” comprises refluxnephropathy, renal shrinkage, rejection and interstitial \\
fibrosis/tubular atrophy & & \\
\hline
\end{tabular}

\section{Serum creatinine}

Figure 1 shows the time course of creatinine in patients with or without acute rejection. The values between these groups were significantly different on each postoperative day $(p<0.05)$. Patients in the NRej group showed a median value of 384 (IQR 307) $\mu \mathrm{mol} / \mathrm{l}$ on postoperative day 1 and stabilised around $130(80) \mu \mathrm{mol} / \mathrm{l}$ upon day 5. Patients in the Rej group had 576 (231) $\mu \mathrm{mol} / \mathrm{l}$ on day 1 and still 195 (185) $\mu \mathrm{mol} / \mathrm{l}$ on day 14.

Fig. 1. Time course of serum creatinine in NRej and Rej group (grey).

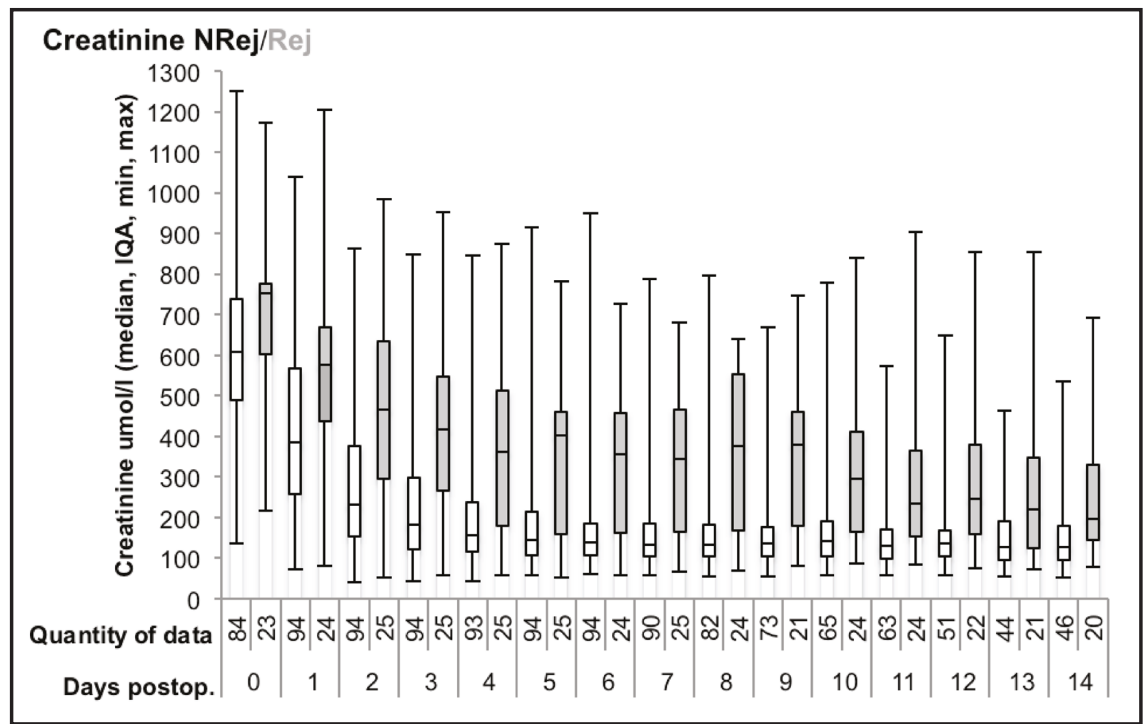

\section{Diuresis}

The NRej group showed a linear decline of diuresis from $3571( \pm 1939) \mathrm{ml} / \mathrm{d}$ to $2424( \pm 826) \mathrm{ml} / \mathrm{d}$ in the second week. The Rej group showed lower diuresis at the first postoperative day $(2947( \pm 1579) \mathrm{ml} / \mathrm{d})$ and in the second week $2045( \pm 1092) \mathrm{ml} / \mathrm{d}$. Differences between NRej and Rej groups were significant on day 3, 4, 5 and $7(\mathrm{p}<0.05)$.

Urine osmolality and osmolaluria

Urine osmolality of the Rej group was lower (366 $\pm 48 \mathrm{mOms} / \mathrm{kg}$ on days 2-8) compared with the NRej group (399 $\pm 80 \mathrm{mOsm} / \mathrm{kg}$ on days 2-8), but difference was significant just 


\section{Kidney Blood Pressure Research}

Fig. 2. Osmolaluria in NRej/Rej.

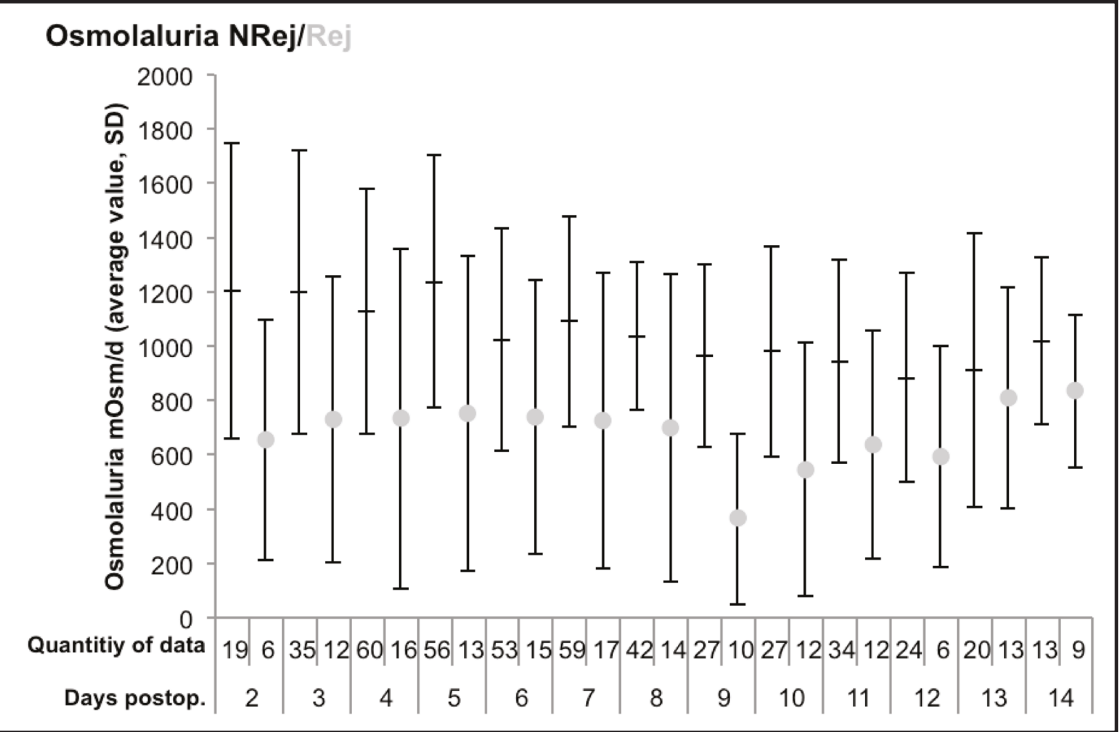

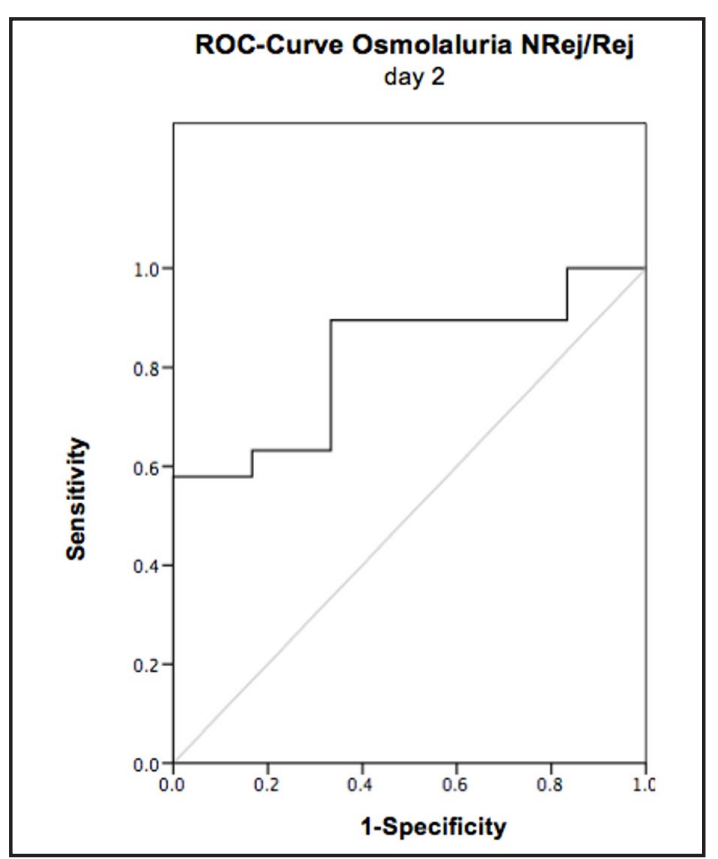

Fig. 3. ROC-Curve for Osmolaluria concerning rejection on day 2. on day $3(\mathrm{p}=0.043), 5(\mathrm{p}=0.031)$ and 8 $(p=0.034)$. Osmolaluria was significantly different between NRej and Rej group on each postoperative day $(\mathrm{p}<0.05)$. Patients without rejection had an osmolaluria of $1204( \pm 544)$ $\mathrm{mOsm} / \mathrm{d}$ on day 2. Thereafter, osmolaluria decreased steadily to $912( \pm 504) \mathrm{mOsm} / \mathrm{d}$ on day 13. Patients with acute rejection in the follow-up had an osmolaluria of 656 $( \pm 443) \mathrm{mOsm} / \mathrm{d}$ on day 2 and $811( \pm 408)$ $\mathrm{mOsm} / \mathrm{d}$ on day 13 (fig 2). The ROC curve of osmolaluria to predict a rejection in the first 14 postoperative days showed an AUC (area under the curve) of 0.816 on day 2 (fig 3 ). If osmolaluria falls below $600 \mathrm{mOsmol} / \mathrm{l}$, sensitivity and specificity for prediction of rejection is $66.7 \%$ and $89.5 \%$, respectively. Setting limit value at $1249 \mathrm{mOsm} / \mathrm{d}$, sensitivity and negative predictive value for rejection were $100 \%$.

Including only patients with histologically confirmed rejection within the first 10 days $(n=19)$, AUC was 0.827 on day 3. At $750 \mathrm{mOsm} / \mathrm{d}$, sensitivity and specificity

was $83.3 \%$ and $79 \%$, respectively. Comparison of osmolaluria in patients who had a biopsy with no proof of rejection (meaning patients with clinical suspicion of rejection; BxNRej, $\mathrm{n}=13$ ) and patients with histologically confirmed rejection could be made only after day 4 because of data restriction. In this clinical relevant situation, AUC was only 0.578 and thus even lower than in the overall group. Comparison of ROC-values for diuresis and osmolaluria between NRej and Rej group showed a similar AUC on day 3 and 4 with a value around 0.74 and a lower AUC for Diuresis (around 0.66) compared with osmolaluria (around 0.8) on day 2 and 5.

The ROC-curves for serum creatinine and osmolaluria concerning prediction of acute rejection showed only little differences (table 2). 


\section{Kidney \\ Blood Pressure Research}

Table 2. Number of measurement values (NMV), AUC, limit values, sensitivity und specificity of serum creatinine and osmolaluria for Rej when undercut (crea) resp. exceeded (osmo) the limit value at the respective postoperative day

\begin{tabular}{lcccccccc}
\hline Rej/NRej & \multicolumn{2}{c}{ Day 2 } & \multicolumn{2}{c}{ Day 3 } & \multicolumn{2}{c}{ Day 4 } & \multicolumn{2}{c}{ Day 5 } \\
\hline & crea & osmo & crea & osmo & crea & osmo & crea & osmo \\
NMV & $94 / 25$ & $19 / 6$ & $94 / 25$ & $35 / 12$ & $93 / 25$ & $60 / 16$ & $94 / 25$ & $56 / 13$ \\
AUC & 0.724 & 0.816 & 0.742 & 0.733 & 0.741 & 0.73 & 0.757 & 0.78 \\
Limit value & 360 & 600 & 260 & 800 & 270 & 1000 & 250 & 760 \\
( $\mu$ mol/l resp. m0sm/d) & & & & & & & & \\
Sensitivity (\%) & 68 & 66.7 & 76 & 75 & 68 & 81 & 64 & 61 \\
Specificity (\%) & 74.5 & 89.5 & 70 & 77 & 80 & 63.3 & 85 & 86 \\
\hline \multicolumn{2}{l}{ crea: creatinine, osmo: osmolaluria, AUC: Area under the curve } \\
\hline
\end{tabular}

\section{Urine strip test}

Strip test was performed every third or fourth day. pH was between 4.5 and 8 in all measurements. Urine glucose was negative in $85 \%$ of all measurements. Semiquantitative protein values $(1-3+, 1+$ around $30 \mathrm{mg} / \mathrm{dl}, 2+$ around $100 \mathrm{mg} / \mathrm{dl}, 3+\geq 500 \mathrm{mg} / \mathrm{dl})$ decreased over the two postoperative weeks in the NRej and the Rej group (from 2.5 (IQR 1.75)+ resp. $2(2)+$ on day 2 to 1(1) + on day 14). Differences between NRej and Rej group were never significant. Haemoglobin/Myoglobine (Hb/Mb) was positive in 249 of 266 (95\%) measurements and in each patient at least once in the observed period. Nitrite, ketone, urobilinogen and bilirubin were not detectable in any patient. Semiquanititative leucocyte test was negative over the whole period in only $14(12 \%)$ patients.

\section{Proteinuria}

Proteinuria (in g/l and g/d) was obtained from 24-hour urine collection. Measurement was done irregularly and at the times of measurement in less than $1 / 3$ of the cohort. Values decreased over the two weeks and there was no significant difference between NRej and Rej Group, but patients with a rejection showed more often a proteinuria $>0.5 \mathrm{~g} / \mathrm{l}$ (fig. 4). Proteinuria $>0.5 \mathrm{~g} / \mathrm{l}$ and $>0.8 \mathrm{~g} / \mathrm{l}$ have a specificity of $80 \%$ and $90 \%$, respectively, regarding prediction of rejection.

Fig. 4. Proteinuria in $\mathrm{g} / \mathrm{l}$ in NRej and Rej groups.

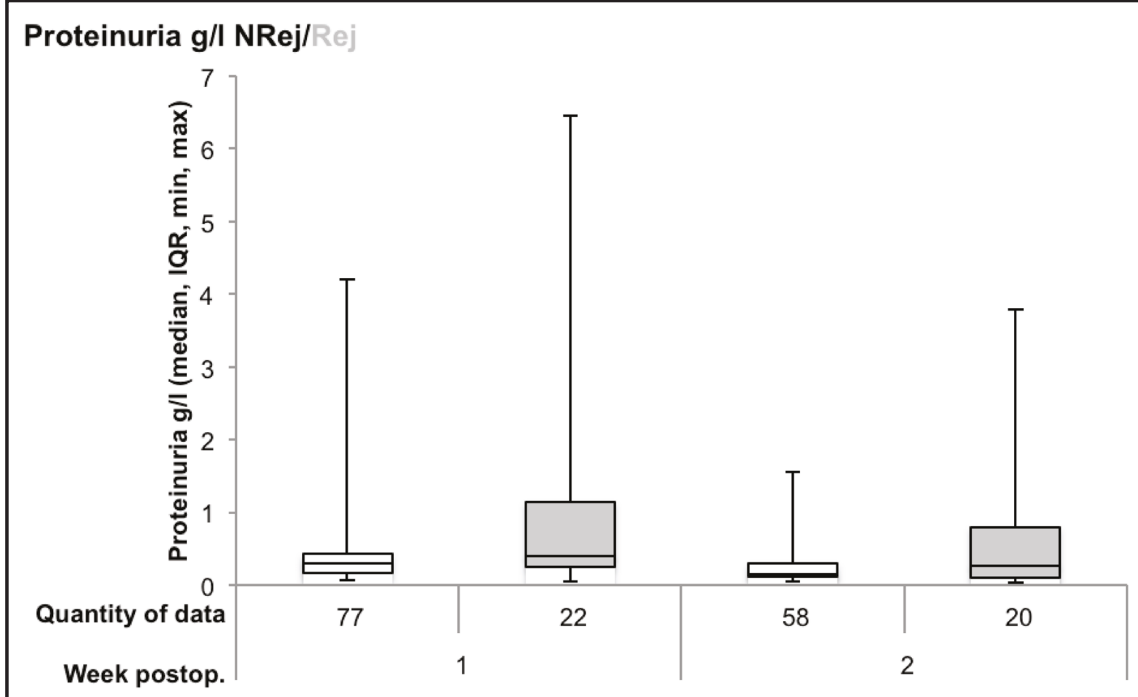

Glucosuria

Glucosuria increased in the NRej group from a median of 1.53 (IQR 7.04) mmol/l in the first week to 2.23 (7.09) $\mathrm{mmol} / \mathrm{l}$ in the second week. In the Rej group, the increase was from 1.23 (4.26) $\mathrm{mmol} / \mathrm{l}$ to 2.91 (7.28) $\mathrm{mmol} / \mathrm{l}$ (not significant). 


\section{Kidney Blood Pressure Research}

Table 3. Number of patients with bacteriuria/infection, with contaminated urine and without bacteria detection
Kidney Blood Press Res 2013;37:116-123

\begin{tabular}{l|l}
\hline DOI: 10.1159/000350065 & (C) 2013 S. Karger AG, Basel
\end{tabular}

Published onlıne: April 13, 2013

www.karger.com/kbr

\begin{tabular}{lcc}
\hline & NRej $(\mathrm{n}=95)$ & Rej $(\mathrm{n}=25)$ \\
\hline Not detectable & $27(28 \%)$ & $4(16 \%)$ \\
Contamination & $28(29 \%)$ & $8(32 \%)$ \\
Asymptomatic bacteriuria/UTI & $30(32 \%)$ & $11(44 \%)$ \\
Percentage values correspond to the proportion of the according \\
group to all patients. UTI: Urinary tract infection \\
\hline
\end{tabular}

\section{Microbiology}

Depending on bacteriological findings a classification into 1) not detectable, 2) contaminated and 3) bacteria found, was performed (table 3). Contamination was defined as $\geq 3$ different bacteria or $\leq 10^{4}$ colony forming units (CFU) $/ \mathrm{ml}$.

Antibiotic prophylaxis was effected with a single-dose of $2.2 \mathrm{~g}$ amoxicilline/clavulanic acid intraoperatively and with co-trimoxazole (sulfamethoxazole/trimethoprim) postoperatively for 6 months. An antibiogram was done, if e.coli, klebsiella, pseudomonas, proteus mirabilis or coagulase negative staphylococci (CNS) were isolated $(n=20)$. All tested bacteria were resistant to co-trimoxazole, 5 additionally to amoxicilline/clavulanic acid. One extended spectrum beta-lactamase producing germ was found. Bacteria resistant to cotrimoxazole were resistant also to ampicillin with one exception. Resistance to tetracycline was also frequent $(70 \%)$.

\section{Discussion}

As urine is easy to obtain and classical parameters of urinalysis are inexpensive, it would be interesting if this method would be able to assist in determining rejection reaction in renal transplants. Osmolality marks the renal concentrating power, which depends on tubular function of the nephrons. In case of an ischemic renal lesion, osmolality is lower compared to a healthy kidney [4]. In this study, osmolality showed significant differences between rejection (Rej) and non-rejection (NRej) group only on some days, which might be due to our low case number. For osmolaluria, we found a more evident difference, but as a parameter to predict rejection osmolaluria did show only little and clinical not significant differences to diuresis regarding the ROC-values. Comparing patients with biopsy but without rejection to patients with histologically confirmed rejection in terms of osmolaluria, the AUC of 0.578 revealed, that it is not possible to distinct between rejection and other complications like tubular necrosis, based on osmolaluria values. The ROC-curves for osmolaluria (on day 2 to 5) and for serum creatinine between NRej and Rej group showed only little differences. Combination of creatinine and osmolaluria measurements did not improve validity regarding prediction of rejection (data not shown). In conclusion, osmolaluria has a similar value as diuresis for prediction of rejection and both parameters do not add benefit to the measurement of serum creatinine alone.

Strip test and microscopic sediment did not show significant differences between Rej and NRej patients and do not qualify to evaluate for a rejection, but may indicate urinary tract infection, polyomavirus nephropathy [2] or a relapse of the underlying disease.

A late occurrence of proteinuria after renal transplantation is associated with reduced graft and patient survival [5]. Reasons include relapse or de novo glomerulonephritis as well as transplant glomerulopathy, chronic rejection, nephrosclerosis, renal vein thrombosis and reflux nephropathy $[6,7]$. Proteinuria is also a risk factor for cardiovascular morbidity and mortality in the general population $[8,9]$. Former studies demonstrated that occurrence of proteinuria in the early phase after renal transplantation is frequent but often not persistent and quantity as well as duration has no prognostic relevance $[10,11]$. However, from the third month after transplantation onwards, even low-level proteinuria $(0.15-0.5 \mathrm{~g} / \mathrm{d})$ is associated with reduced graft and patient survival [12]. Consistent with these results we 


\section{Kidney \\ Blood Pressure Research}

did not remark a prognostic relevance for measurement of proteinuria per day regarding rejection reaction. For proteinuria per litre no significant difference could be found either but proteinuria $>0.5 \mathrm{~g} / \mathrm{l}$ occurred more often in the Rej group. Despite all constrictions, we do recommend weekly measurements of proteinuria, which might provide indications for relapse of underlying disease, renal vein thrombosis or rejection.

For glucosuria no significant difference between the groups were detected. As diagnosis of diabetes mellitus demands blood glucose measurements anyway, there is no role for the measurement of glucosuria after renal transplantation.

Antibiotic prophylaxis is done intra- and postoperative due to the operative intervention, the immunosuppression and the ureteral catheter. Antibiotic prophylaxis as used in our center prevents effectively symptomatic and asymptomatic bacteriuria as well as other infections like Pneumocystis jirovecii-pneumonia, toxoplasmosis, brucellosis, listeriosis and nocardiosis [13]. However, no consistent recommendations for screening and treatment of asymptomatic bacteriuria exist until now [14]. Results regarding the impact of urinary tract infections and bacteriuria on graft and patient survival are contradictory. While some recommend screening and treatment of asymptomatic bacteriuria in the first six months post renal transplantation as they found an impact between urinary tract infections and patient and graft survival [15], others suppose restricting antibiotic treatments for asymptomatic bacteriuria in the absence of pyuria, occurring later than one month post transplantation to be safe [16]. In a meta-analysis no significant difference was seen in graft loss comparing antibiotic prophylaxis with placebo for 1 month during the first 6 months after renal transplantation. Prophylaxis lowered the risk for developing sepsis and bacteriuria, but no effects on graft survival could be demonstrated [17]. As in the majority of our patients no bacteria have been isolated, we assume that antibiotic prophylaxis was effective in these cases. Asymptomatic bacteriuria and UTI were diagnosed in $35 \%(n=41)$ of the transplant recipients. As expected, all cases with antibiogram $(n=29)$ showed resistance to co-trimoxazole. As $60 \%$ of the cultures were stated negative, we suggest performing a semiquantitative agar plate test first. Positive samples are to be sent for further microbiological examination. With this approach, remarkable costs can be saved.

\section{Conflict of Interests}

The authors declare that they have no conflicts.

\section{References}

1 Grunewald R, Fiedler G, Stock B, Grunewald J, Müller G: Immunocytological determination of lymphocytes and monocytes/macrophages in urinary sediments of renal allograft recipients. Nephrol Dial Transplant 2000;15:888-892.

2 Bubendorf L, Mihatsch M: [Urinary cytology after renal transplantation]. Ther Umsch 2006;63:609-614.

-3 Zweig M, Campbell G: Receiver-operating characteristic (ROC) plots: a fundamental evaluation tool in clinical medicine. Clin Chem 1993;39:561-577.

4 Zimmermann-Spinnler M: Urinlabor. Medical Laboratory Consulting AG Liestal CH 1991. S41- 44.

-5 Fernández-Fresnedo G, Plaza J, Sánchez-Plumed J, Sanz-Guajardo A, Palomar-Fontanet R, Arias M: Proteinuria: a new marker of long-term graft and patient survival in kidney transplantation. Nephrol Dial Transplant 2004;19:iii47-51.

-6 Peddi V, Dean D, Hariharan S, Cavallo T, Schroeder T, First M: Proteinuria following renal transplantation: correlation with histopathology and outcome. Transplant Proc 1997;29:101-103. 


\section{Kidney \\ Blood Pressure Research}

7 Vathsala A, Verani R, Schoenberg L, Lewis RM, Van Buren CT, Kerman RH, Kahan BD: Proteinuria in cyclosporine-treated renal transplant recipients. Transplantation 1990;49:35-41.

8 Grimm RJ, Svendsen K, Kasiske B, Keane W, Wahi M: Proteinuria is a risk factor for mortality over 10 years of follow-up. MRFIT Research Group. Multiple Risk Factor Intervention Trial. Kidney Int Suppl 1997;63:S10-14.

-9 Kannel W, Stampfer M, Castelli W, Verter J: The prognostic significance of proteinuria: the Framingham study. Am Heart J 1984;108:1347-1352.

-10 Pérez Fontán M, Rodríguez-Carmona A, García Falcón T, Valdés F: Early proteinuria in renal transplant recipients treated with cyclosporin. Transplantation 1999;67:561-568.

-11 Yildiz A, Erkoç R, Sever MS, Türkmen A, Ecder ST, Türk S, Kiliçarslan I, Ark E : The prognostic importance of severity and type of post-transplant proteinuria. Clin Transplant 1999;13:241-244.

12 Cherukuri A, Welberry-Smith MP, Tattersall JE, Ahmad N, Newstead CG, Lewington AJ, Baker RJ: The clinical significance of early proteinuria after renal transplantation. Transplantation 2010;89:200-207.

-13 Nicolle L: Asymptomatic bacteriuria: review and discussion of the IDSA guidelines. Int J Antimicrob Agents 2006;28:S42-48.

14 Nicolle L, Bradley S, Colgan R, Rice J, Schaeffer A, Hooton T: Infectious Diseases Society of America guidelines for the diagnosis and treatment of asymptomatic bacteriuria in adults. Clin Infect Dis 2005;40:643-654.

15 Yacoub R, Akl NK: Urinary tract infections and asymptomatic bacteriuria in renal tranpslant recipients. J Glob Infect Dis 2011;3:383-389.

16 El Amari EB, Hadaya K, Bühler L, Berney T, Rohner P, Martin PY, Mentha G, van Delden C: Outcome of treated and untreated asymptomatic bacteriuria in renal transplant recipients. Nephrol Dial Transplant 2011;26:4109-4114.

17 Green H, Rahamimov R, Gafter U, Leibovitci L, Paul M: Antibiotic prophylaxis for urinary tract infections in renal transplant recipients: a systematic review and meta-analysis. Transpl Infect Dis 2011;13:441-447. 